\title{
Coexistence Performance of IEEE 802.15.4 Wireless Sensor Networks Under IEEE 802.11b/g Interference
}

\author{
Wei Yuan - Xiangyu Wang • \\ Jean-Paul M. G. Linnartz • Ignas G. M. M. Niemegeers
}

Published online: 13 December 2011

(C) The Author(s) 2011. This article is published with open access at Springerlink.com

\begin{abstract}
IEEE 802.15.4 Wireless Sensor Networks (WSNs) and IEEE 802.11b/g Wireless Local Area Networks (WLANs) are often collocated, causing a coexistence issue since these networks share the same $2.4 \mathrm{GHz}$ Industrial, Scientific, and Medical band. In our previous work, we built a coexistence model of IEEE 802.15.4 WSNs and IEEE 802.11b/g WLANs. By identifying three distinct coexistence regions, the model explained the coexistence behavior of IEEE 802.15.4 WSNs and IEEE 802.11b/g WLANs, and the model was experimentally validated. In this paper, we improve the model by introducing two important implementation factors: the transceiver's Rx-to-Tx turnaround time and the Clear Channel Assessment partial detection effect. The enhanced model significantly improves the accuracy on explaining and predicting the coexistence performance of IEEE 802.15.4 WSNs in the real-life environment. Furthermore, under the guidance of the model, the coexistence performance of IEEE 802.15.4 WSNs is extensively investigated in various coexistence scenarios by analysis, simulation and experiments, respectively. The simulation and experimental results agree with our analysis. The coexistence model is believed to be helpful in resolving the coexistence issue.
\end{abstract}

This work was partially supported by the Dutch Freeband PNP 2008 project.

W. Yuan $(\varangle) \cdot$ X. Wang $\cdot$ J.-P. M. G. Linnartz

Philips Research, High Tech Campus 37, Eindhoven, The Netherlands

e-mail: mailtoyw@gmail.com

X. Wang

e-mail: xiangyu.wang@philips.com

W. Yuan · I. G. M. M. Niemegeers

Delft University of Technology, Delft, The Netherlands

I. G. M. M. Niemegeers

e-mail: i.niemegeers@ewi.tudelft.nl

J.-P. M. G. Linnartz

Eindhoven University of Technology, Eindhoven, The Netherlands

e-mail: j.p.linnartz@philips.com 
Keywords IEEE 802.15.4 - IEEE 802.11b - IEEE 802.11g - ZigBee · Coexistence · Coexistence region - Interference · Clear channel assessment - Energy detection · Unfairness · Rx-to-Tx turnaround time $\cdot$ CCA partial detection

\section{Introduction}

As a low-power and low-cost technology, IEEE 802.15.4 is becoming an enabler for the emerging Wireless Sensor Networks (WSNs). Like IEEE 802.11b/g Wireless Local Area Networks (WLANs), IEEE 802.15.4 WSNs also operate in the same 2.4 GHz Industrial, Scientific, and Medical (ISM) band. Because of their complimentary applications, IEEE 802.15.4 WSNs and IEEE $802.11 \mathrm{~b} / \mathrm{g}$ WLANs are often collocated within an interfering range of each other and therefore their ability to coexist needs to be investigated.

There have been some studies about the coexistence between IEEE 802.15.4 WSNs and IEEE 802.11b/g WLANs. According to [1,2,4], IEEE 802.15.4 WSNs have little impact on IEEE 802.11 WLANs performance. IEEE 802.11 WLANs, however, may have a serious impact on IEEE 802.15.4 WSNs performance if the channel allocation is not carefully taken into account [1,3]. While the conclusion is true in general, these studies dealt with only limited coexistence scenarios. For example, in [3], the Packet Error Rate (PER) of IEEE 802.15.4 WSNs under IEEE 802.11b interference was analyzed based on an assumption of blind transmissions, i.e., both IEEE 802.11b and IEEE 802.15.4 nodes can transmit packets freely regardless of whether the channel state is busy or not. However, it was shown that this assumption holds in only one of three coexistence regions defined in [5], i.e., a region in which neither IEEE 802.15.4 nodes nor IEEE 802.11b/g nodes can sense the other, but IEEE 802.15.4 nodes could still suffer from IEEE 802.11b/g interference. The other two coexistence regions are a region in which IEEE 802.15.4 nodes and IEEE $802.11 \mathrm{~b} / \mathrm{g}$ nodes can sense each other and a region in which IEEE 802.15.4 nodes can sense IEEE $802.11 \mathrm{~b} / \mathrm{g}$ nodes, but not vice versa. These regions are further addressed in Sect. 3.

Yuan et al. [5] presented a coexistence model of IEEE 802.15.4 WSNs and IEEE $802.11 \mathrm{~b} / \mathrm{g}$ WLANs. Although the model depicted the coexistence behavior well in general, owing to not considering some implementation factors, it failed to precisely explain some coexistence performances in the real-life environment. In [6], the model was experimentally validated, but only very limited quantitative analysis was given.

In this paper, by introducing two important implementation factors: the transceiver's Rxto-Tx turnaround time and the Clear Channel Assessment (CCA) partial detection effect, we improve the analytical model, which significantly enhances the model's accuracy on explaining and predicting the coexistence performance of IEEE 802.15.4 WSNs under IEEE $802.11 \mathrm{~b} / \mathrm{g}$ interference in the real-life environment. Furthermore, under the guidance of the model, the coexistence performance of IEEE 802.15.4 WSNs under IEEE 802.11b/g interference is extensively investigated in all of three coexistence regions and in different scenarios by analysis, simulation and experiments, respectively.

The remainder of the paper is organized as follows: Sect. 2 gives an overview of the IEEE 802.11b/g and IEEE 802.15.4 standard. Section 3 presents the improved coexistence model to characterize the coexistence issue. Under the guidance of the improved model, the performance analysis of IEEE 802.15.4 WSNs under IEEE $802.11 \mathrm{~b} / \mathrm{g}$ interference is given in Sect. 4. Section 5 shows the evaluation of the improved model and the coexistence performances in various scenarios are investigated in Sect. 6. Conclusions are drawn in Sect. 7. 


\section{IEEE 802.11b/g and IEEE 802.15.4 Overview}

In this section, we give a brief overview about the MAC sublayers of IEEE $802.11 \mathrm{~b} / \mathrm{g}$ and IEEE 802.15.4, with relevant details on CCA modes.

\subsection{IEEE $802.11 \mathrm{~b} / \mathrm{g}$}

The IEEE 802.11b and IEEE 802.11g standards define the Medium Access Control (MAC) sublayer and the Physical (PHY) layer for WLANs. Both standards operate at 13 overlapping channels in the $2.4 \mathrm{GHz}$ ISM band and the bandwidth of each channel is $22 \mathrm{MHz}$. The IEEE 802.11b/g MAC employs the Carrier Sense Multiple Access with Collision Avoidance (CSMA/CA) mechanism. CCA is used in the physical layer to determine the channel occupancy [8]. CCA performs Energy Detection (ED), or Carrier Sense (CS), or a combination of the two, i.e., CCA shall report a busy channel upon detection of any energy above the ED threshold, or a signal with the known features, e.g., the modulation and spreading characteristics, or a known signal with energy above the ED threshold. Owing to involving only integrating the square of the received signal if implemented in the analog domain or summing squares of its samples in the digital domain, ED is a universal mechanism that can be deployed in all systems without requiring any knowledge of the type of underlying modulation scheme employed at the physical layer [9]. Therefore, in a heterogenous network environment, only ED can, though unreliably in some cases [9], sense the channel occupancy of other types of networks. Since we address the coexistence issue of a heterogenous network environment in this paper, ED is always assumed as the only employed CCA mode.

Before initiating a transmission, an IEEE $802.11 \mathrm{~b} / \mathrm{g}$ node senses a channel using either ED or CS (or both) to check whether it is busy because of transmissions by other nodes. If the channel is sensed idle for a Distributed coordination function Inter-Frame Space (DIFS) time interval the node will transmit a packet. Otherwise, the node defers its transmission. As the channel becomes idle for a DIFS time interval, the node will generate a random backoff delay based on an integer uniformly chosen in a Contention Window $(\mathrm{CW})$, i.e., $[0, W]$, where $W$ is the size of the CW. The backoff timer decreases by one as long as the channel is sensed idle for a backoff time slot. The backoff counter will be frozen when a transmission is detected on the channel, and resumed when the channel is sensed idle again for a DIFS interval. When the backoff timer counts down to zero, the node transmits a packet. Immediately after receiving a packet correctly, the destination node waits for a Short Inter Frame Spacing (SIFS) interval and then sends an ACK back to the source node. If the source node receives the ACK, the size of CW remains the same value; otherwise, it doubles.

\subsection{IEEE 802.15.4}

The IEEE 802.15.4 standard defines the MAC sublayer and the PHY layer. Its operational frequency bands include the $2.4 \mathrm{GHz}$ ISM band. There are two versions of IEEE 802.15.4 CSMA/CA: slotted and unslotted. In this paper, we discuss only the popular unslotted one. Like IEEE 802.11b/g WLANs, IEEE 802.15.4 WSNs also employ CSMA/CA for the medium access control. In IEEE 802.15.4 WSNs, the channel is sensed only during a CCA period rather than during both a CCA and a backoff period like in IEEE 802.11b/g WLANs. The standard specifies that either ED or CS (or both) is used to check the channel state, but does not provide precise algorithms, e.g., for combing multiple samples within the CCA in case of the digital ED receiver, and thus the algorithms differ from implementation to implementation. If the channel is sensed busy during the CCA period, the size of CW in 
IEEE 802.15.4 WSNs doubles, and when the number of the channel access attempts exceeds macMaxCSMABackoffs [10], the maximum number of backoffs the CSMA-CA algorithm will attempt before declaring a channel access failure, the pending packet is discarded.

\section{A Coexistence Model of IEEE 802.11 b/g and IEEE 802.15.4 Networks}

In this work, saturated IEEE $802.11 \mathrm{~b} / \mathrm{g}$ interference is always assumed, which means there is always an IEEE $802.11 \mathrm{~b} / \mathrm{g}$ packet available for transmission. This corresponds to the presence of the worst-case of interference, which in practice would occur, e.g., as IEEE $802.11 \mathrm{~b} / \mathrm{g}$ nodes transfer video streams or large files.

Under IEEE $802.11 \mathrm{~b} / \mathrm{g}$ interference, an IEEE 802.15 .4 packet can be successfully received if either of the following two conditions is satisfied [5].

Condition A: When the IEEE 802.15.4 packet overlaps an IEEE $802.11 \mathrm{~b} / \mathrm{g}$ packet, the in-band interference power from the IEEE $802.11 \mathrm{~b} / \mathrm{g}$ packet is significantly lower than the useful signal power from the IEEE 802.15.4 packet at an IEEE 802.15.4 receiver. According to the IEEE 802.15.4 specification [10], if interference is so weak that the in-band signal-tointerference ratio (SIR) is larger than 5-6 dB, an IEEE 802.15.4 packet can be successfully received with a probability of $99 \%$.

Condition B: The transmission time of an IEEE 802.15.4 packet is shorter than the interframe idle time between two consecutive IEEE $802.11 \mathrm{~b} / \mathrm{g}$ packets so that the IEEE 802.15.4 packet does not overlap an IEEE $802.11 \mathrm{~b} / \mathrm{g}$ packet.

Our coexistence model includes power and timing aspects, which are presented as follows:

(1) Power Aspect: The transmit powers of IEEE $802.11 \mathrm{~b} / \mathrm{g}$ nodes and IEEE 802.15.4 nodes are typically $100 \mathrm{~mW}$ [8] and $1 \mathrm{~mW}$ [10], respectively. In case of comparable CCA thresholds, the significant difference in the transmit power can result in three distinct regions as illustrated in Fig. 1:

R1: a region in which IEEE 802.15.4 nodes and IEEE 802.11b/g nodes can sense each other;

R2: a region in which IEEE 802.15.4 nodes can sense IEEE 802.11b/g nodes, but not vice versa;

R3: a region in which neither can sense the other, but IEEE 802.15.4 nodes could still suffer from IEEE $802.11 \mathrm{~b} / \mathrm{g}$ interference.

(2) Timing Aspect:

Fig. 1 Coexistence regions of IEEE 802.15.4 and IEEE $802.11 \mathrm{~b} / \mathrm{g}$

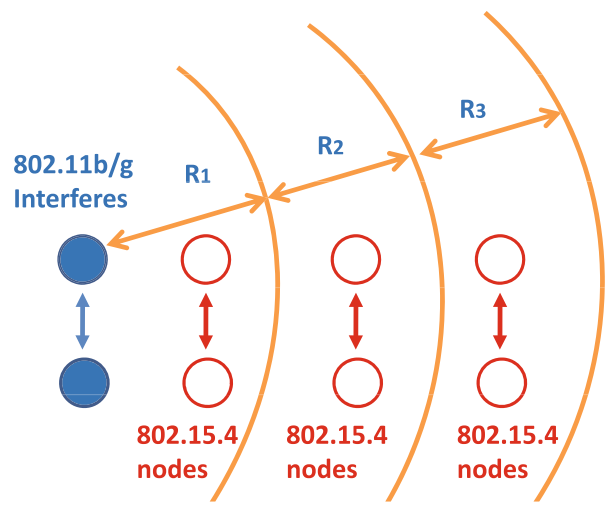


Table 1 IEEE 802.15.4 and IEEE 802.11b/g system parameters and additional parameters used in simulation and experiments

\begin{tabular}{llll}
\hline & IEEE 802.15 .4 & IEEE $802.11 \mathrm{~b}$ & IEEE $802.11 \mathrm{~g}$ \\
\hline Transmit power & $0 \mathrm{dBm}$ & $17 \mathrm{dBm}$ & $17 \mathrm{dBm}$ \\
Receiver sensitivity & $-85 \mathrm{dBm}$ & $-76 \mathrm{dBm}$ & $-82 \mathrm{dBm}$ \\
Bandwidth & $2 \mathrm{MHz}$ & $22 \mathrm{MHz}$ & $22 \mathrm{MHz}$ \\
Data rate & $250 \mathrm{kbps}$ & $11 \mathrm{Mbps}$ & $54 \mathrm{Mbps}$ \\
Backoff unit $T_{b s}$ & $320 \mu \mathrm{s}$ & $20 \mu \mathrm{s}$ & $9 \mu \mathrm{s}$ \\
SIFS & $192 \mu \mathrm{s}$ & $10 \mu \mathrm{s}$ & $10 \mu \mathrm{s}$ \\
DIFS & $\mathrm{N} / \mathrm{A}$ & $50 \mu \mathrm{s}$ & $28 \mu \mathrm{s}$ \\
CCA duration & $128 \mu \mathrm{s}$ & $\leq 15 \mu \mathrm{s}$ & $\leq 4 \mu \mathrm{s}$ \\
CCA threshold & $-85 \mathrm{dBm}$ & $-84 \mathrm{dBm}$ & $-84 \mathrm{dBm}$ \\
CW & 7 & 31 & 15 \\
Center frequency & $2410 \mathrm{MHz}$ & $2412 \mathrm{MHz}$ & $2412 \mathrm{MHz}$ \\
Payload size & $30 \mathrm{bytes}$ & $1500 \mathrm{bytes}$ & $1500 \mathrm{bytes}$ \\
ACK & No & Yes & Yes \\
Transmit intensity & Every $20 \mathrm{~ms}$ & Saturated & Saturated \\
Tx-to-Rx turnaround & $<192 \mu \mathrm{s}$ & $<10 \mu \mathrm{s}$ & $<10 \mu \mathrm{s}$ \\
Rx-to-Tx turnaround & $<192 \mu \mathrm{s}$ & $<5 \mu \mathrm{s}$ & $<5 \mu \mathrm{s}$ \\
\hline
\end{tabular}

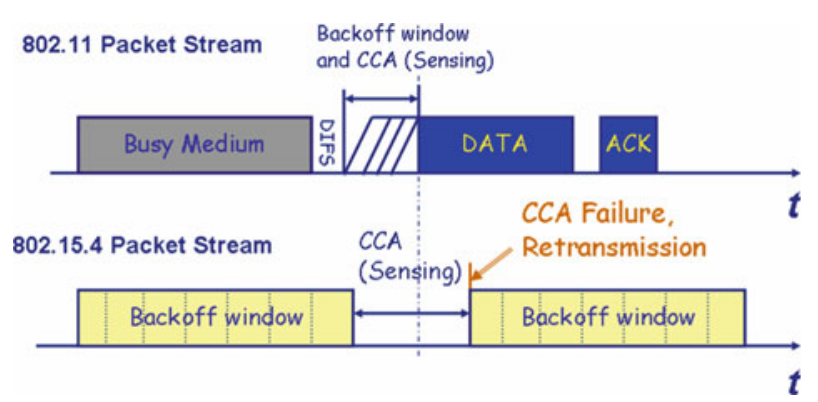

Fig. 2 In R1: the shorter timing gives IEEE $802.11 \mathrm{~b} / \mathrm{g}$ nodes priority over IEEE 802.15 .4 nodes to access the channel and therefore causes unfairness to the IEEE 802.15.4 nodes

\section{- In R1}

In R1, an IEEE 802.11b/g node and an IEEE 802.15.4 node can sense each other by ED and therefore both of their CSMA/CA mechanisms work, i.e. as one is transmitting, the other has to be waiting. IEEE 802.15.4 nodes, however, typically have a 10-30 times longer timing than IEEE 802.11b/g nodes, e.g., the backoff slot unit is 320, 20 and $9 \mu$ s for IEEE 802.15.4, IEEE $802.11 \mathrm{~b}$ and IEEE $802.11 \mathrm{~g}$, respectively, shown in Table 1. The shorter timing gives IEEE $802.11 \mathrm{~b} / \mathrm{g}$ nodes priority over IEEE 802.15 .4 nodes to access the channel and therefore causes unfairness to the IEEE 802.15.4 nodes in R1, as illustrated in Fig. 2.

Once the IEEE 802.15.4 nodes sense the channel idle for a CCA duration and therefore seize the channel, they can transmit packets, theoretically, free from interference because the IEEE $802.11 \mathrm{~b} / \mathrm{g}$ nodes will defer for the IEEE 802.15.4 packet transmission in this region, i.e. R1. In practice, however, the IEEE 802.15.4 nodes have to spend the maximum 12 symbol periods, i.e. $192 \mu \mathrm{s}$ at most, on turning around their states from receiving to transmitting, i.e., 


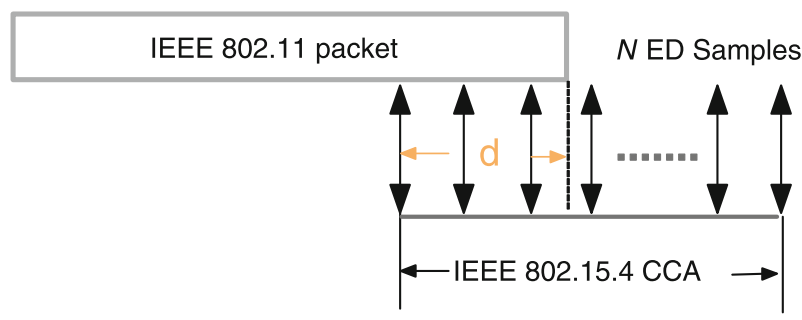

Fig. 3 IEEE 802.15.4 ED detects only a partial IEEE 802.11 b packet over a CCA duration

an Rx-to-Tx turnaround time [10], during which the channel state may change from idle to busy, though.

Besides, in many research papers and widely used simulation tools like OPNET, it is often ignored and therefore implicitly assumed that a CCA always reports a busy channel once a CCA window has an overlap to any extent with a transmitting packet. In practice, however, this is not true. A typical digital ED receiver samples the channel $N$ times during the CCA, sums up the sampled energy and compares the sum, $E_{\text {samples }}$, to a preset ED threshold $\Gamma$. If $E_{\text {samples }}>\Gamma$, ED reports the channel busy; otherwise, it reports the channel idle. It is not uncommon that ED samples only a part of a packet, i.e. the overlapping part, denoted as $d$, shown in Fig. 3. We call this effect $\boldsymbol{C C A}$ partial detection. We define a specific overlap duration, denoted as $d_{m}$, such that given a $\Gamma, d_{m}$ equals the maximum $d$ over which $E_{\text {samples }} \leq \Gamma$.

Thus, under the saturated IEEE 802.11b/g interference in R1, IEEE 802.15.4 nodes could seize the channel and transmit packets if

$$
\mathrm{CCA}-d_{m} \leq t_{\text {idle }}
$$

where $t_{\text {idle }}$ is the idle time between two consecutive IEEE $802.11 \mathrm{~b} / \mathrm{g}$ packets. According to the specification [8],

$$
t_{i d l e} \triangleq \mathrm{DIFS}+t_{b o}=\mathrm{DIFS}+m \cdot T_{b s}
$$

where $t_{b o}$ is a random period of time for an additional deferral time before transmitting and $t_{b o} \triangleq m \cdot T_{b s}$, where $T_{b s}$ is a backoff unit and $m$ is a random integer drawn from a uniform distribution over the interval $\left[0, \mathrm{CW}_{\min }\right]$. Note that Eq. (2) does not include the turnaround time of Rx-to-Tx and Tx-to-Rx for IEEE $802.11 \mathrm{~b} / \mathrm{g}$ nodes since it is very short $(<15 \mu \mathrm{s}$ in total [8]). By contrast, the turnaround time, $T_{t a}$, of Rx-to-Tx and Tx-to-Rx for IEEE 802.15.4 nodes should be taken into account because it could be even longer than an IEEE 802.15.4 CCA duration. The values of these parameters are shown in Table 1.

In practice, satisfying inequality (1) can only ensure IEEE 802.15.4 nodes seize the channel and transmit packets but not guarantee the transmitted packets free from IEEE $802.11 \mathrm{~b} / \mathrm{g}$ interference, which additionally requires either

$$
\mathrm{CCA}+T_{t a}-d_{m} \leq t_{\text {idle }}
$$

or a constantly high SINR at the IEEE 802.15.4 receivers.

From the discussion above, we learn that the practical CCA implementation has a significant impact on the performance of CCA, causing CCA performs in practice not as "perfect" as described in theory. In Sect. 5, we will further investigate the real CCA performance in more details. 


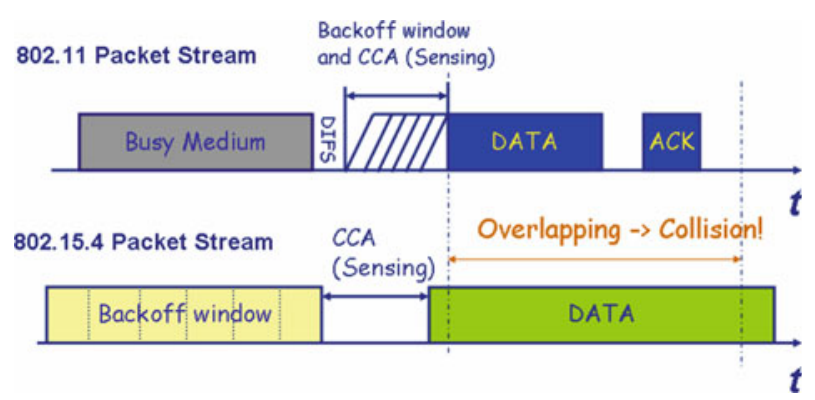

Fig. 4 In R2, IEEE 802.11b/g nodes fails to sense IEEE 802.15.4 nodes

\section{- In R2}

In R2, IEEE 802.15.4 nodes can sense IEEE $802.11 \mathrm{~b} / \mathrm{g}$ nodes but not vice versa in case of comparable CCA thresholds, because the transmit power of IEEE $802.11 \mathrm{~b} / \mathrm{g}$ nodes is much higher than that of IEEE 802.15.4 nodes. Consequently, when IEEE 802.11b/g nodes are transmitting, IEEE 802.15.4 nodes have to be waiting, whereas when IEEE 802.15.4 nodes are transmitting, IEEE $802.11 \mathrm{~b} / \mathrm{g}$ nodes are not aware and thus simply proceed to transmit, probably causing an overlapping in packet transmissions, as shown in Fig. 4.

Therefore, to check whether IEEE 802.15.4 nodes can have successful transmissions in $\mathrm{R} 2$, we first see whether non-overlapping transmissions are possible. Like in R1, inequality (1) needs to be satisfied. In addition, since IEEE $802.11 \mathrm{~b} / \mathrm{g}$ nodes do not defer anymore for IEEE 802.15.4 packets in R2, to ensure non-overlapping transmissions, the following condition also needs to be satisfied:

$$
\mathrm{CCA}+T_{t a}-d_{m}+t_{p}+\mathrm{SIFS}+\mathrm{ACK} \leq t_{i d l e}
$$

where $t_{p}$ is the transmission time of an IEEE 802.15.4 packet, and SIFS and ACK are those of IEEE 802.15.4. According to the parameter values given in Table 1, however, this condition cannot be satisfied. Thus, in case of using ACK, for successful transmissions of IEEE 802.15.4 packets in R2, the power condition A in Sect. 3 has to be satisfied. In case of not using ACK, the condition (4) becomes

$$
\mathrm{CCA}+T_{t a}-d_{m}+t_{p} \leq t_{i d l e}
$$

This condition can hardly be satisfied unless IEEE 802.15.4 packets are very short, e.g., $t_{p}=512 \mu \mathrm{s}$ (corresponding to 16-byte packets transmitted at the rate of $250 \mathrm{kbps}$ ), given that $t_{\text {idle }}=670 \mu$ s (i.e., $m=\mathrm{CW}_{\min }=31, T_{b s}=20 \mu \mathrm{s}$ in Eq. (2)) and CCA $=128 \mu \mathrm{s}$.

\section{- In R3}

In R3, neither IEEE 802.15.4 nodes nor IEEE $802.11 \mathrm{~b} / \mathrm{g}$ nodes can sense the other. IEEE 802.15.4 nodes, however, may still suffer from the IEEE $802.11 \mathrm{~b} / \mathrm{g}$ interference in case of weak IEEE 802.15.4 links, because a range in which a wireless device can cause interference to others is usually larger than that where it can be sensed by the others. This means both IEEE 802.15.4 nodes and IEEE 802.11b/g nodes can freely transmit packets without deferring for the other, which is described as an assumption called blind transmissions in [3].

Like in R2, it can be shown that in case of using ACK, the condition for non-overlapping transmission can never hold in R3, whereas it could hold in case of not using ACK and 
very short transmitted packets. In both cases, the successful transmissions of IEEE 802.15.4 packets could happen if the power condition $A$ in Sect. 3 is satisfied.

\section{Performance Analysis of IEEE 802.15.4 WSNs Under IEEE 802.11b/g Interference}

We have done a performance analysis of IEEE 802.15.4 WSNs under IEEE 802.11b/g interference in [5]. However, the analysis there does not take into account the Rx-to-Tx turnaround time and the CCA partial detection as addressed above, which may have a significant effect. Besides, the analysis was limited only to the coexistence region R1. Also, only one performance metric, i.e., throughput, is derived there. In this paper, we will consider those factors, extend the analysis to all the three coexistence regions, and derive the other two important performance metrics, i.e., packet loss ratio and packet delay, in addition to throughput. Moreover, we will propose two important concepts: inhibition loss and collision loss.

For ease of analysis, we assume that there is only one pair of IEEE 802.15.4 nodes and one pair of IEEE $802.11 \mathrm{~b} / \mathrm{g}$ nodes. In each pair, one node is a transmitter and the other is a receiver. Moreover, the physical channel condition is ideal. According to $[1,2,4]$ and our simulation, IEEE 802.15.4 has little impact on the IEEE 802.11 performance, which suggests us to assume that the IEEE $802.11 \mathrm{~b} / \mathrm{g}$ traffic is not affected by the IEEE 802.15 .4 traffic. Thus, the IEEE $802.11 \mathrm{~b} / \mathrm{g}$ transmitter can always receive ACKs after transmitting data packets, keeping its contention window equal to the initial value, i.e., $\mathrm{CW}_{\min }$. Finally, we assume that IEEE $802.11 \mathrm{~b} / \mathrm{g}$ traffic is in the saturation mode, which means that there is always at least one packet awaiting transmission at the transmitter.

As shown in Fig. 5, for each transmission attempt, an IEEE 802.15.4 node performs a backoff first for an interval sampled from a uniform integer distribution over $\left[0,2^{B E_{i}}-1\right](i=$ $0,1,2,3,4)$, where $B E_{i}$ is the backoff exponent for the $i$ th retransmission attempt, where the 0th retransmission attempt means the first transmission attempt. A successful CCA will be followed by an IEEE 802.15.4 packet transmission. Otherwise, in case of a busy channel, the IEEE 802.15.4 node will defer for a backoff period defined by $B E_{i+1}$ and then perform a CCA again until the default maximum retry limit, i.e. 4, is reached [10], where an error of channel access failure will be reported to the upper layer. In either case, a new transmission cycle will start with a backoff period defined by $B E_{0}$ for the next packet to be transmitted.

To obtain the IEEE 802.15.4 network performance metrics such as packet loss ratio, throughput and packet delay, we need to get two key probabilities, $p_{i}$ and $p_{c}$, where $p_{i}$ is the probability that the channel is idle over an IEEE 802.15.4 CCA duration and $p_{c}$ is the probability that the transmitted IEEE 802.15 .4 packets are hit by IEEE $802.11 \mathrm{~b} / \mathrm{g}$ interference. Let us first derive $p_{i}$ and $p_{c}$, and then the performance metrics in R1, R2 and R3 respectively.

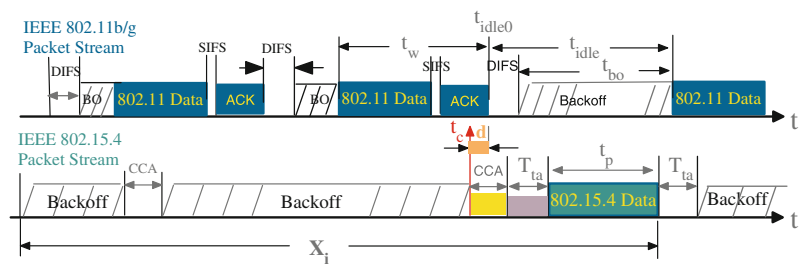

Fig. 5 Coexistence model in timing aspect 


\subsection{Coexistence Performance in R1}

Owing to the assumption that the IEEE $802.11 \mathrm{~b} / \mathrm{g}$ traffic is not affected by the IEEE 802.15 .4 traffic, $p_{i}$ is constant. In fact, $p_{i}$ is the equivalent of the probability that $E_{\text {samples }} \leq \Gamma$ as addressed in Sect. 3(2).

Although an IEEE 802.15.4 CCA may start at any point of the IEEE 802.11b/g packet stream, for a successful IEEE 802.15.4 packet transmission, denoted as E, the CCA should start within the interval $\left[t_{i d l e 0}-d_{m}, t_{i d l e 0}+t_{i d l e}-\mathrm{CCA}+d_{m}\right]$, where $t_{\text {idle } 0}$ is the start time of the idle period $t_{i d l e}$. Thus, $p_{i}$ is given by

$$
p_{i}=P\{\mathrm{E}\}=\sum_{m=a-k}^{\mathrm{CW}_{\min }} P\left\{\mathrm{E}_{\mathrm{m}}\right\}
$$

where $E_{m}$ represents $E$, i.e., a successful IEEE 802.15.4 packet transmission, conditioned on the chosen retransmission moment $m$, with $t_{b o}=m T_{b s}, a$ equals 4 and 12 for IEEE 802.11b and IEEE 802.11g nodes, respectively, and $k=\left\lfloor d_{m} / T_{b s}\right\rfloor$.

Furthermore,

$$
\begin{aligned}
P\left\{\mathrm{E}_{\mathrm{m}}\right\}= & P\left\{t_{b o}=m T_{b s}\right\} \\
& \cdot P\left\{t_{i d l e 0}-d_{m} \leq t_{c} \leq t_{i d l e 0}+\mathrm{DIFS}+m T_{b s}-\mathrm{CCA}+d_{m}\right\}
\end{aligned}
$$

where $t_{c}$ is the CCA start time, uniformly distributed over $\left[0, t_{s}\right], t_{s}$ is the transmission cycle time of an IEEE $802.11 \mathrm{~b} / \mathrm{g}$ packet, i.e. $t_{s}=t_{w}+$ DIFS $+m T_{b s}$ and $t_{w}$ is the sum of an IEEE $802.11 \mathrm{~b} / \mathrm{g}$ packet transmission time, a following SIFS period and ACK period, shown in Fig. 5.

Since the backoff time is uniformly distributed, we get

$$
P\left\{t_{b o}=m T_{b s}\right\}=\frac{1}{\mathrm{CW}_{\min }+1}
$$

Besides, as $k=\left\lfloor d_{m} / T_{b s}\right\rfloor$,

$$
\begin{aligned}
P\left\{t_{i d l e 0}-d_{m} \leq t_{c}\right. & \left.\leq t_{i d l e 0}+\mathrm{DIFS}+m T_{b s}-\mathrm{CCA}+d_{m}\right\} \\
& \approx \frac{\mathrm{DIFS}+m T_{b s}-\mathrm{CCA}+2 k T_{b s}}{\mathbb{E}\left[t_{w}\right]+\mathrm{DIFS}+m T_{b s}}
\end{aligned}
$$

Thus, according to (6), (7), (8) and (9), $p_{i}$ is given by

$$
p_{i}=\frac{1}{\mathrm{CW}_{\min }+1} \sum_{m=a-k}^{\mathrm{CW}_{\min }} \frac{\mathrm{DIFS}+m T_{b s}+2 k T_{b s}-\mathrm{CCA}}{\mathbb{E}\left[t_{w}\right]+\mathrm{DIFS}+m T_{b s}}
$$

According to the IEEE 802.15.4 specification [10], a pending IEEE 802.15.4 packet shall be discarded after $M+1$ times channel access failures, where $M$ is the maximum number of backoffs the CSMA-CA algorithm will attempt before declaring a chennel access failure. We call this kind of loss inhibition loss. Thus the inhibition loss probability, denoted as $\alpha$, is given by

$$
\alpha=\left(1-p_{i}\right)^{M+1}
$$

Then $\beta$, the probability of IEEE 802.15 .4 packets which can be sent out, is given by

$$
\beta=1-\alpha
$$


Now we deal with $p_{c}$, the probability that an IEEE 802.15.4 packet, though sent out by a transmitter, collides with an IEEE $802.11 \mathrm{~b} / \mathrm{g}$ packet. As the collision is due to an overlapping of the IEEE 802.15 .4 packet and the IEEE $802.11 \mathrm{~b} / \mathrm{g}$ packet, let us get the probability, $p_{n o}$, that a transmitted IEEE 802.15.4 packet does not overlap (hence not collide) with an IEEE $802.11 \mathrm{~b} / \mathrm{g}$ packet. Actually, $p_{n o}$ is the equivalent of the probability that a CCA plus a followed Rx-to-Tx turnaround time $T_{t a}$ fall into the period $\left[t_{i d l e 0}-d_{m}, t_{i d l e 0}+t_{i d l e}\right]$ as shown in Fig. 5. This is because in such a case, IEEE 802.11 nodes will be able to sense the coming IEEE 801.15.4 packet and therefore suspend the transmission of their own packets. Thus, similar to the derivation of $p_{i}, p_{n o}$ is given by

$$
p_{n o}=\frac{1}{\mathrm{CW}_{\min }+1} \sum_{n=b-k}^{\mathrm{CW}_{\text {min }}} \frac{\mathrm{DIFS}+n T_{b s}+2 k T_{b s}-\mathrm{CCA}-T_{t a}}{\mathbb{E}\left[t_{w}\right]+\mathrm{DIFS}+n T_{b s}}
$$

where $b=\left\lceil\left(\mathrm{CCA}+T_{t a}\right) / T_{b s}\right\rceil$, which equals 14 and 33 for IEEE 802.11b and IEEE 802.11g, respectively, given the default $192 \mu \mathrm{s}$ of $T_{t a}$. Since the IEEE $802.11 \mathrm{~g} \mathrm{CW}_{\min }$ is only 15 , less than 33, Eq. (13) cannot hold in case of IEEE 802.11g given our assumption that the size of the contention window stays at $\mathrm{CW}_{\min }$. Thus, $p_{n o}=0$ for IEEE $802.11 \mathrm{~g}$ in our case.

Then $p_{c}$ can be given by

$$
p_{c}=\beta \cdot\left(1-\frac{p_{n o}}{p_{i}}\right) \cdot p_{e}
$$

where $p_{e}$ is the IEEE 802.15.4 packet error rate. Assuming that bit errors are independent, $p_{e}$ is given by

$$
p_{e}=1-\left(1-p_{b}\right)^{N}
$$

where $p_{b}$ is the IEEE 802.15.4 Bit Error Rate (BER) and $N$ is the number of bits of an IEEE 802.15.4 packet. According to equation [10], $p_{b}$ is given by

$$
p_{b}=\frac{8}{15} \times \frac{1}{16} \times \sum_{r=2}^{16}(-1)^{r}\left(\begin{array}{c}
16 \\
r
\end{array}\right) e^{\left(20 \times \operatorname{SINR} \times\left(\frac{1}{r}-1\right)\right)}
$$

Thus, $p_{c}$ can be computed by Eqs. (12), (13), (14), (15), (16).

With $p_{i}$ and $p_{c}$, we now derive throughput $S$, packet loss ratio $\eta$ and expected packet delay $\mathbb{E}\left(t_{d}\right)$, respectively. Owing to the assumption that the IEEE $802.11 \mathrm{~b} / \mathrm{g}$ traffic is not affected by the IEEE 802.15.4 traffic and the fact that the timing of IEEE 802.11b/g and IEEE 802.15.4 is significantly different, the transmission cycle times of IEEE 802.15.4 packets are considered independent of each other. Therefore, the transmission of IEEE 802.15.4 packets is essentially a renewal process. Let $X$ denote the transmission cycle time of a packet, which either is transmitted successfully at the $i$ th retransmission or fails to be transmitted eventually after the $M+1$ unsuccessful channel access attempts, where $M$ is the maximum number of backoffs the CSMA-CA algorithm will attempt before declaring a channel access failure. The default $M$ is 4 [10]. Therefore, $X$ is actually the inter-renewal time of the renewal process. Furthermore, let $\{W(t) ; t>0\}$ be a renewal reward function for the renewal process with expected value of the inter-renewal time $\mathbb{E}(X)$.

Thus according to [11], the IEEE 802.15.4 throughput $S$ is given by

$$
S=\lim _{t \rightarrow \infty} \frac{1}{t} \int_{\tau=0}^{t} W(\tau) d \tau=\frac{\mathbb{E}\left[W_{m}\right]}{\mathbb{E}[X]} \quad \text { with probability } 1
$$


where $\mathbb{E}\left[W_{m}\right]$ is the expected value of the reward, which is either the packet size, $t_{p}$, or zero, depending on whether a packet is sent out during the $m$ th renewal interval and whether the packet is received successfully. Therefore,

$$
\begin{aligned}
\mathbb{E}\left[W_{m}\right] & =\left(1-p_{c}\right)\left[\mathbb{E}\left[t_{p}\right] p_{i} \sum_{m=0}^{M}\left(1-p_{i}\right)^{m}+0 \cdot\left(1-p_{i}\right)^{M+1}\right] \\
& =\left(1-p_{c}\right) \mathbb{E}\left[t_{p}\right] p_{i} \sum_{m=0}^{M}\left(1-p_{i}\right)^{m}
\end{aligned}
$$

where $\mathbb{E}\left[t_{p}\right]$ is the expected $t_{p}$. We now compute $\mathbb{E}[X]$. In case of the saturated IEEE 802.15.4 traffic, let

$$
A=\sum_{m=0}^{M}\left[p_{i}\left(1-p_{i}\right)^{m}\left[\sum_{n=0}^{m} \mathbb{E}\left[B_{n}\right]+(m+1) \mathrm{CCA}+2 T_{t a}+\mathbb{E}\left[t_{p}\right]\right]\right]
$$

we can get

$$
\mathbb{E}(X)=A+\left(1-p_{i}\right)^{M+1}\left[\sum_{n=0}^{M} \mathbb{E}\left[B_{n}\right]+(M+1) \mathrm{CCA}\right]
$$

where $\mathbb{E}\left[B_{n}\right]$ is the expected backoff time $B_{n}$ for the $n$th retransmission. By substituting Eqs. (18), (20) into Eq. (17), the IEEE 802.15.4 throughput $S$ is obtained.

Note that in case of the non-saturated IEEE 802.15.4 traffic, the expected inter-renewal time is different from the one computed in Eq. (20). For example, for a traffic with a constant packet interval time $T>\mathbb{E}(X)$ in Eq. (20), the throughput $S=\mathbb{E}\left[W_{n}\right] / T$.

The IEEE 802.15.4 packet loss consists of two kinds of losses: inhibition loss and collision loss. Therefore, the IEEE 802.15.4 packet loss ratio $\eta$ is given by

$$
\eta=\alpha+p_{c}
$$

The expected channel access delay $\mathbb{E}\left(t_{d}\right)$ includes only the delay between packet arrival and the start of its first transmission attempt. For those packets that fail to seize a transmission opportunity, the contribution to $\mathbb{E}\left(t_{d}\right)$ is set to zero, even though a retry at upper protocol layers may cause a large delay. Thus, $\mathbb{E}\left(t_{d}\right)$ is computed by

$$
\mathbb{E}\left(t_{d}\right)=\sum_{m=0}^{M} p_{i}\left(1-p_{i}\right)^{m}\left[\sum_{n=0}^{m} \mathbb{E}\left[B_{n}\right]+(m+1) \mathrm{CCA}+T_{t a}\right]
$$

\subsection{Coexistence Performance in R2}

In R2, IEEE 802.15.4 nodes can still sense IEEE $802.11 \mathrm{~b} / \mathrm{g}$ traffic. Therefore, $p_{i}$ stays the same as in R1 and so does the inhibition loss probability $\alpha$. Since IEEE $802.11 \mathrm{~b} / \mathrm{g}$ nodes cannot sense an IEEE 802.15.4 packet any more in R2, for avoiding an overlapping transmission, inequality (4) or (5) needs to be satisfied, which is almost impossible as addressed in Sect. 3. Thus, $p_{c}=\beta \cdot p_{e}$. The throughput $S$ can also be given by Eqs. (17), (18) and (20). 
The packet loss ratio $\eta$ and the expected packet delay $\mathbb{E}\left(t_{d}\right)$ are given by Eqs. (21) and (22), respectively.

\subsection{Coexistence Performance in R3}

In R3, $p_{i}=1$ and therefore the inhibition loss probability $\alpha=0$. Thus, $p_{c}=p_{e}$. The throughput $S$ is given by Eq. (17), where $\mathbb{E}\left(W_{m}\right)=\left(1-p_{e}\right) \cdot \mathbb{E}\left(t_{p}\right)$ and $\mathbb{E}(X)=\mathbb{E}\left(B_{0}\right)+$ $\mathrm{CCA}+2 T_{t a}+\mathbb{E}\left(t_{p}\right)$. The packet loss ratio $\eta=\alpha+p_{c}=p_{c}$ and the expected packet delay $\mathbb{E}\left(t_{d}\right)=0$.

\section{Evaluation of the Improved Coexistence Model}

In order to evaluate our enhanced analytical model in a nearly real-world environment, we carried out a number of experiments using off-the-shelf hardware. In some cases, OPNET simulation results are also provided as a reference.

\subsection{Experimental Testbed and Configuration}

In [6], we designed and set up a compact testbed as shown in Fig. 6, which included the following items:

- two IEEE 802.11b nodes (Linksys WRT54G - in the 802.11b mode): a Tx and an Rx;

- two IEEE 802.15.4 nodes (AquisGrain [12]);

- two RF shielded isolation boxes;

- one attenuator matrix box;

- two PCs with testing software.

The antennas of IEEE 802.11b nodes and IEEE 802.15.4 nodes were connected by cables via the attenuator matrix, the attenuation values of which can be adjusted to emulate various physical distances in a wireless environment. To isolate from other RF interference, IEEE 802.15.4 nodes were put into the RF shielded isolation boxes such that we got a controlled RF environment, allowing the measurements to be repeatable.

A functional diagram of the testbed is depicted in Fig. 7. The attenuation losses among those nodes are as follows,

- $x_{1}$ : between IEEE 802.11b Tx and IEEE 802.15.4 Tx;

- $x_{2}$ : between IEEE 802.11b Rx and IEEE 802.15.4 Tx;

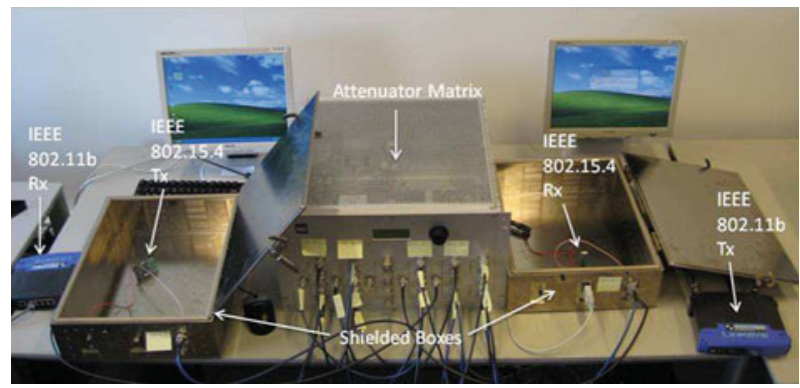

Fig. 6 Testbed of the coexistence model of IEEE 802.11b and IEEE 802.15.4 networks 


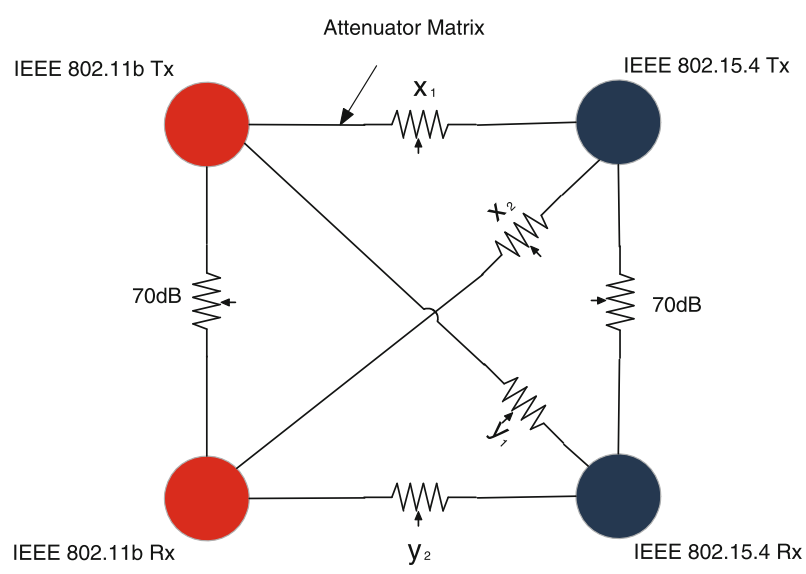

Fig. 7 Functional diagram of the coexistence testbed

- $y_{1}$ : between IEEE 802.11b Tx and IEEE 802.15.4 Rx;

- $y_{2}$ : between IEEE 802.11b Rx and IEEE 802.15.4 Rx.

$x_{1}, x_{2}, y_{1}$ and $y_{2}$ are adjustable, from 32 to $212 \mathrm{~dB}$, which are the minimum and the maximum attenuation loss respectively we can make given the testbed. Moreover, we set both the attenuation losses between IEEE 802.11b Tx and Rx and between IEEE 802.15.4 Tx and Rx at $70 \mathrm{~dB}$, so that the two links have a very good quality, i.e., the packet loss ratio of the IEEE 802.15.4 link is close to zero and the throughput of the IEEE $802.11 \mathrm{~b}$ link is $6.82 \mathrm{Mbps}$, the maximum value achievable in our case given the parameter values used in the experiments as shown in Table 1.

In the experiments, the IEEE 802.15.4 Tx constantly sent only broadcast packets and the IEEE 802.15.4 Rx did not send any packets including ACKs. The IEEE 802.11b Tx generated a saturated packet stream and the IEEE $802.11 \mathrm{~b}$ Rx sent ACKs only. Moreover, we made the IEEE 802.11b Tx and the Rx had the same impact on the IEEE 802.15.4 Tx and on the IEEE 802.15.4 Rx, respectively. We therefore always set the same values for $x_{1}$ and $x_{2}$, and $y_{1}$ and $y_{2}$, respectively. For the sake of brevity, we let $x=x_{1}=x_{2}$ and $y=y_{1}=y_{2}$.

- R1: Given the IEEE 802.15.4 transmit power of $0 \mathrm{dBm}$ and the IEEE 802.11b CCA threshold of $-84 \mathrm{dBm}$, when $x \geq 84 \mathrm{~dB}$, the IEEE $802.11 \mathrm{~b}$ nodes will not be able to sense the IEEE 802.15.4 nodes, i.e., R1 is the region where $x<84 \mathrm{~dB}$.

- R3: Although the IEEE $802.11 \mathrm{~b}$ transmit power is $17 \mathrm{dBm}$, only $16.9 \%$ falls into the $2 \mathrm{MHz}$ band of IEEE 802.15.4 [3], i.e., 9.3 dBm. Given the CCA threshold of $-85 \mathrm{dBm}$, the IEEE 802.15.4 nodes will not be able to sense the IEEE 802.11 b nodes when $x \geq 94.3 \mathrm{~dB}$, i.e., $\mathrm{R} 3$ is the region where $x \geq 94.3 \mathrm{~dB}$.

- R2: By definition, $\mathrm{R} 2$ is in between $\mathrm{R} 1$ and $\mathrm{R} 3$. Therefore, $\mathrm{R} 2$ is the region where $84 \mathrm{~dB}$ $<x<94.3 \mathrm{~dB}$.

\subsection{Evaluation of the Improved Coexistence Model}

We now carry out the experiments to identify these regions. For convenience, we start with identifying R1, followed by R3 and R2. 


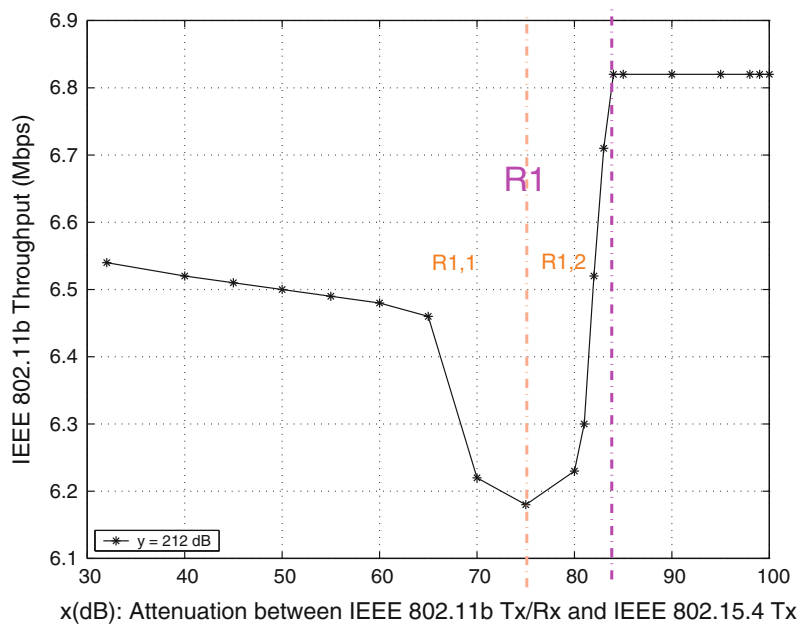

Fig. 8 In R1: IEEE 802.11 b/g nodes can also sense IEEE 802.15.4 traffic

\subsubsection{R1 Identification}

To identify R1 and to investigate details of the coexistence behavior of IEEE $802.11 \mathrm{~b}$ and IEEE 802.15.4 networks, we measure the IEEE 802.11b throughput and the IEEE 802.15.4 packet loss ratio in the following two cases:

- $\quad \mathbf{y}=212$ dB (inhibition loss only): Given such a high attenuation loss, the IEEE 802.11b Tx and Rx have actually no impact on the IEEE 802.15.4 Rx but only on the Tx. Therefore, in this case, the IEEE 802.15.4 packet loss is not due to collision but due to inhibition only, i.e. $p_{e}=0$ and therefore $p_{c}=0$ by Eq. (14). As the IEEE 802.15.4 Rx does not send any packets including ACKs in our experiments, only the IEEE 802.15.4 Tx could affect the throughput of the IEEE $802.11 \mathrm{~b}$ network. Thus, we can adjust only $x$ to observe the impact of the IEEE 802.15.4 Tx on the IEEE 802.11b Tx and Rx.

As an example, in Fig. 8, we can see that as $x=32 \mathrm{~dB}$, the IEEE 802.11b throughput is approximately $6.54 \mathrm{Mbps}$, less than its maximum, i.e., $6.82 \mathrm{Mbps}$, which suggests that the IEEE 802.11 b network is suffering, though not very seriously, from the IEEE 802.15.4 traffic.

As $x$ increases, we expected the IEEE $802.11 \mathrm{~b}$ throughput to increase as well because of the weakening IEEE 802.15.4 Tx impact. However, we surprisingly found in Fig. 8 that as $x$ increases until about $75 \mathrm{~dB}$, the IEEE $802.11 \mathrm{~b}$ throughput actually decreases, which suggests that the impact of the IEEE 802.15.4 Tx on the IEEE 802.11b network increases rather than decreases. This is confirmed by Fig. 9, in which we can see that for $32 \mathrm{~dB}<x<80 \mathrm{~dB}$, as $x$ increases, the IEEE 802.15.4 CCA failure rate decreases, which suggests that more IEEE 802.15.4 packets were sent out indeed and the impact of the IEEE 802.15.4 Tx on the IEEE $802.11 \mathrm{~b}$ network therefore increases. The explanation we have for this is that as $x$ increases, the missed probability of the IEEE 802.15.4 ED increases and consequently, more often the IEEE 802.15.4 Tx senses the channel idle and sends out more packets than it should, which lowers the channel occupancy of the IEEE $802.11 \mathrm{~b}$ traffic and thus the throughput of the IEEE 802.11b network. As addressed in [9], with a high missed probability, ED is not a reliable CCA method. Especially, as the detected signal weakens, the missed probability of ED goes even higher. 


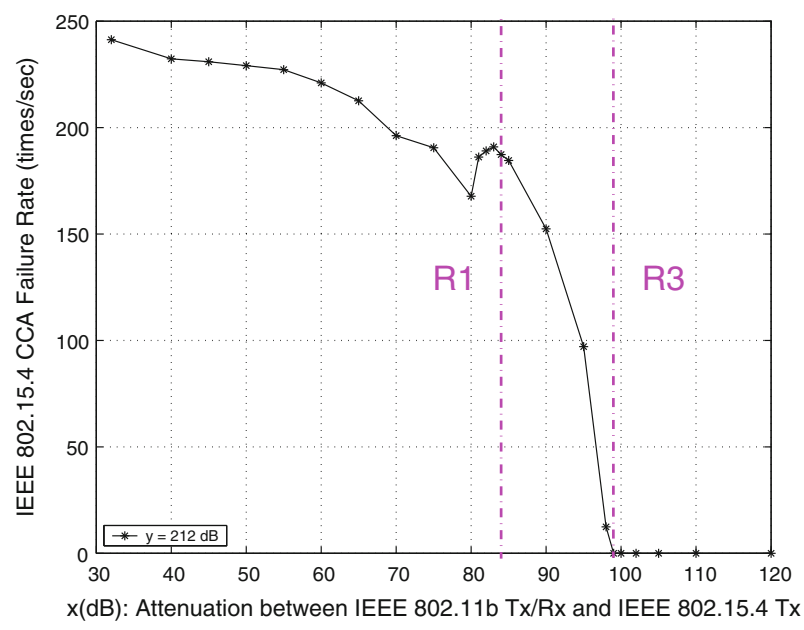

Fig. 9 IEEE 802.15.4 Tx CCA Failure Rate

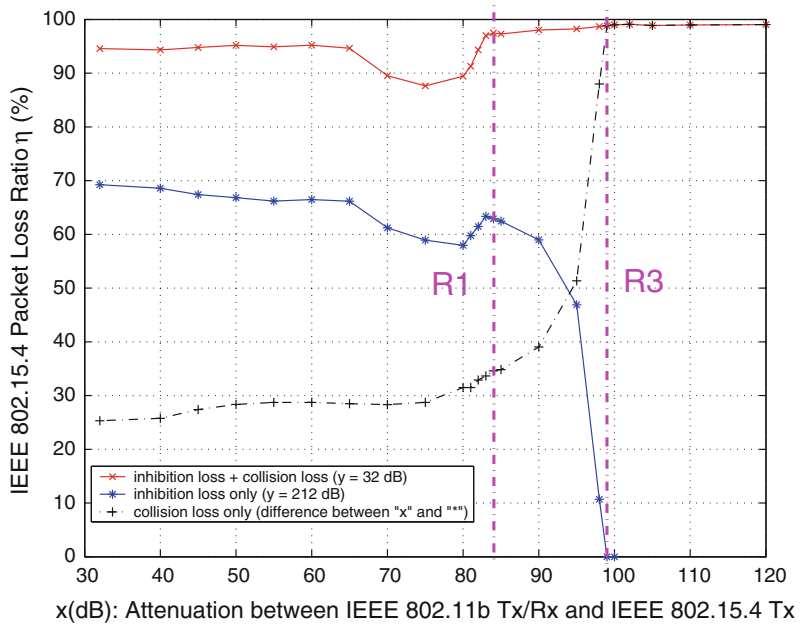

Fig. 10 In R3: neither can sense the other, but IEEE 802.15.4 nodes could still suffer from IEEE $802.11 \mathrm{~b} / \mathrm{g}$ interference

In Fig. 8, for $75 \mathrm{~dB}<x<84 \mathrm{~dB}$, as $x$ increases, which suggests that the influence from the IEEE 802.15.4 Tx is getting less. This is because the IEEE 802.11b Tx/Rx are leaving the region where they are able to sense the IEEE 802.15.4 Tx.

For $x \geq 84 \mathrm{~dB}$, as $x$ increases, the IEEE $802.11 \mathrm{~b}$ throughput stays constant at its maximum, i.e., $6.82 \mathrm{Mbps}$, suggesting that the IEEE $802.11 \mathrm{~b} \mathrm{Tx} / \mathrm{Rx}$ are not able to sense the IEEE 802.15.4 Tx and are not affected by the IEEE 802.15.4 Tx anymore. On the other hand, from the Fig. 10 we see that in the region of $x<84 \mathrm{~dB}$, the IEEE 802.15.4 Tx has a high packet loss ratio, which suggests it can sense IEEE $802.11 \mathrm{~b}$ traffic there. We therefore conclude that the region where $x<84 \mathrm{~dB}$ is $\mathrm{R} 1$. 


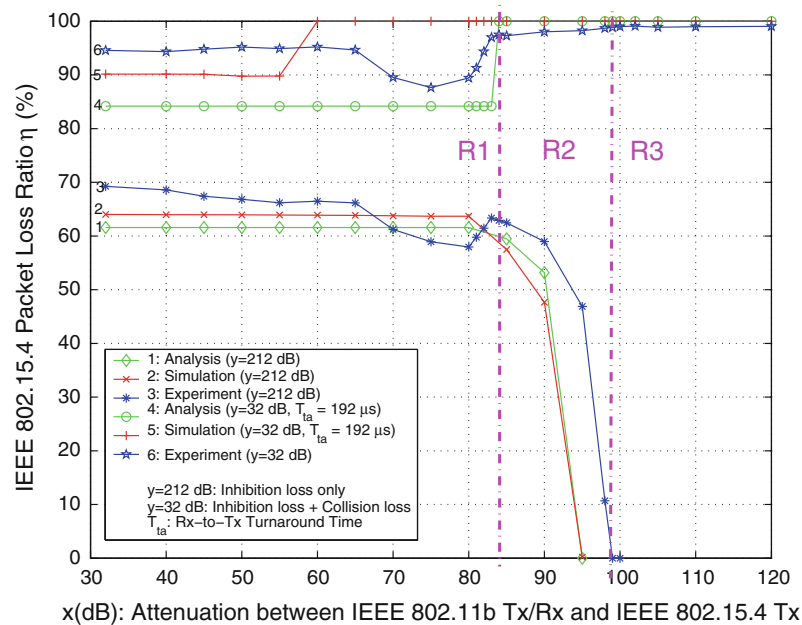

Fig. 11 Analysis, simulation and experimental results for the performance of an IEEE 802.15.4 WSN under IEEE $802.11 \mathrm{~b} / \mathrm{g}$ interference

We may further divide R1 into two subregions as R1,1 $(\mathrm{x}<75 \mathrm{~dB})$ and $\mathrm{R} 1,2(75 \mathrm{~dB}<$ $x<84 \mathrm{~dB}$ ), illustrated in Fig. 8. R1,2 is the transition region, where the IEEE $802.11 \mathrm{~b}$ Tx is leaving the region in which it is able to sense the IEEE 802.15.4 nodes.

Note that the curve representing the case of "inhibition loss only $(y=212 \mathrm{~dB})$ " in Fig. 10 is not monotonic. We see that when $x \geq 80 \mathrm{~dB}$, there is a "hump", i.e., the IEEE 802.15.4 packet loss ratio goes up first until $x=83 \mathrm{~dB}$ and then goes down again to zero at $x=98 \mathrm{~dB}$. The "hump" is because the IEEE 802.11b Tx and Rx are leaving R1, as shown in Fig. 8, and therefore getting less influence from the IEEE 802.15.4 traffic, which results in more IEEE $802.11 \mathrm{~b}$ packets sent out and therefore more IEEE 802.15.4 channel access failures. For $x \geq 83 \mathrm{~dB}$, as $x$ increases, although more IEEE $802.11 \mathrm{~b}$ packets are sent out, these packets cause only decreasing IEEE 802.15.4 channel access failures owing to their weakening power. For $x \geq 98 \mathrm{~dB}$, the IEEE 802.15 .4 packet loss ratio equals zero, which means that IEEE 802.15.4 Tx cannot sense IEEE $802.11 \mathrm{~b}$ traffic anymore and therefore does not suffer from the channel access failures. This is confirmed in Fig. 9, where we can see that the IEEE 802.15.4 CCA failure stays zero for $x \geq 98 \mathrm{~dB}$.

It is worthy noting that according to [1,2,4], IEEE 802.15.4 WSNs has little impact on the IEEE 802.11 WLANs performance. This conclusion is true in general, but in some cases, IEEE 802.15.4 WSNs may have a non-negligible impact on the performance of IEEE $802.11 \mathrm{~b} / \mathrm{g}$ WLANs. For example, in Fig. 8, we see that for $70 \mathrm{~dB}<x<80 \mathrm{~dB}$, the IEEE $802.11 \mathrm{~b}$ throughput is about $6.2 \mathrm{Mbps}$, approximately $10 \%$ less than its maximum, i.e., 6.82 Mbps. In case of weaker IEEE 802.11b links and heavier IEEE 802.15.4 traffic, the IEEE $802.11 \mathrm{~b}$ throughput is supposed to be even lower.

In Sect. 4, we derived the packet loss ratio of IEEE 802.15.4 WSNs under IEEE 802.11b/g interference. To validate our analysis, we put the analytical results and the experimental results together in Fig. 11, which also includes an OPNET simulation result as a reference. We can see that in case of $y=212 \mathrm{~dB}$ (inhibition loss only), the analytical, the OPNET simulation and the experimental results have a good match in general. Some small mismatches in details, e.g., the $3.7 \mathrm{~dB}$ difference in the lower-bound of R3 between the analytical value $(94.3 \mathrm{~dB})$ 
and the experimental value $(98 \mathrm{~dB})$, may be attributed to the errors in the measurement and/or the hardware implementation.

Although R1 has been identified, to reveal more insights about the impact from the IEEE 802.11b traffic on the IEEE 802.15.4 network, we further measured the IEEE 802.15.4 packet loss ratio in the following case.

- $\quad \mathbf{y}=32 \mathrm{~dB}$ (inhibition loss + collision loss): In this case, the IEEE 802.11b Tx and Rx influence not only the IEEE 802.15.4 Tx but also the IEEE 802.15.4 Rx. Consequently, the IEEE 802.15.4 packet loss includes not only the inhibition loss but also the collision loss. Note that given $\mathrm{y}=32 \mathrm{~dB}$, the IEEE $802.11 \mathrm{~b}$ Tx and $\mathrm{Rx}$ impact on the IEEE 802.15.4 $\mathrm{Rx}$ is so strong that SINR $<-45 \mathrm{~dB}$, which suggests $p_{e}=1$ and therefore $p_{c}$ depends only on $p_{n o}$ by Eq. (14). The relationship between $x$ and the packet loss ratio $\eta$ is based on Eq. (21), which is shown by the curve of "inhibition loss + collision loss ( $y=32 \mathrm{~dB})$ " in Fig. 11.

Given the detailed discussion about the coexistence behavior of IEEE 802.11b and IEEE 802.15.4 networks above in R1, the identification of R3 and R2 is straightforward as follows.

\subsubsection{R3 Identification}

From the curve of "inhibition loss only $(y=212 \mathrm{~dB}$ )" in Fig. 10, we see that as $x \geq 98 \mathrm{~dB}$, the IEEE 802.15.4 packet loss ratio because of the channel access failures goes down to zero, which means that IEEE 802.15.4 Tx cannot sense IEEE 802.11b traffic and therefore does not suffer from the channel access failures anymore. This is confirmed in Fig. 9, where we can see that the IEEE 802.15.4 CCA failure rate goes down to zero as $x \geq 98 \mathrm{~dB}$. We therefore conclude that in the region where $x \geq 98 \mathrm{~dB}$, neither IEEE 802.15.4 nodes nor IEEE 802.11 b nodes can sense the other, but IEEE 802.15.4 nodes may still suffer from the IEEE $802.11 \mathrm{~b}$ interference, which is exactly what R3 defines. Note that we have calculated that R3 is the region where $x \geq 94.3 \mathrm{~dB}$ rather than $98 \mathrm{~dB}$ as suggested by the experiment. The $3.7 \mathrm{~dB}$ difference may be attributed to the errors in the measurement and/or the hardware implementation, which has been mentioned in Sect. 5.2.1.

\subsubsection{R2 Identification}

For convenience, Fig. 8 is superimposed on Fig. 10, resulting in Fig. 12. We can see that in the region between $\mathrm{R} 1$ and $\mathrm{R} 3$, i.e., $84 \mathrm{~dB}<x<98 \mathrm{~dB}$, there is still some IEEE 802.15.4 packets loss owing to the channel access failures, which suggests in that region, IEEE 802.15.4 Tx can still sense the IEEE $802.11 \mathrm{~b}$ Tx/Rx, while not vice versa. This is exactly the region which R2 defines.

Upon till now, all R1, R2 and R3 have been clearly identified and our coexistence model has been validated by the experiments.

\subsubsection{More Discussions}

In case of $y=32 \mathrm{~dB}$ (inhibition loss + collision loss), we can see from the experimental result shown as the curve 6 in Fig. 11 that the IEEE 802.15.4 packet loss ratio is quite high, even in the region R1, where the IEEE 802.15.4 packet loss ratio is supposed to be low instead because in R1, IEEE 802.15.4 nodes and IEEE 802.11b nodes can hear each other and therefore their CSMA/CA mechanism should be working there. We found out that this is because 


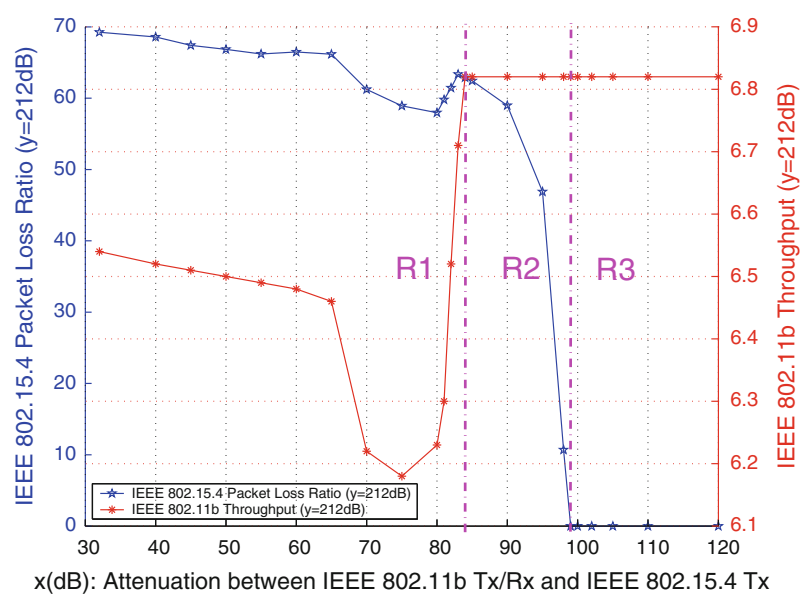

Fig. 12 In R2: IEEE 802.15.4 nodes can sense IEEE 802.11b/g nodes, but not vice versa

in reality, an IEEE 802.15.4 node cannot send out a packet immediately after a successful CCA. Instead, the node has to take an additional time as long as an Rx-to-Tx turnaround time after the CCA to switch its transceiver state from receiving to transmitting. During this Rx-to-Tx turnaround time, however, the channel may become busy again due to the IEEE 802.11b/g traffic, which can cause a collision with a coming IEEE 802.15.4 packet. As such, the effectiveness of CCA gets impaired. Curve 4 and 5 in Fig. 11 show the analytical and the simulative results respectively, given an Rx-to-Tx turnaround time of $192 \mu \mathrm{s}$, the default value specified in the standard [10]. These results are close to the experimental one shown as Curve 6, which suggests the Rx-to-Tx turnaround time in the experiment is around $192 \mu \mathrm{s}$.

To learn that how much IEEE 802.15.4 coexistence performance could deteriorate due to a none-zero Rx-to-Tx turnaround time in reality, we compare curve 1 and curve 2 with curve 3 and curve 6, respectively, in the region R1 of Fig. 11. Curve 1 and curve 2 show the analytical and simulative IEEE 802.15.4 packet loss ratios, respectively, in case of a zero Rx-to-Tx turnaround time, while Curve 3 and curve 6 show the experimental IEEE 802.15.4 packet loss ratios in case of around $192 \mu \mathrm{s}$ Rx-to-Tx turnaround time. We first compare curve 1 and curve 2 to curve 3 , all of which happen in case of no collision loss. We take the case of $x=40 \mathrm{~dB}$ for instance and see the IEEE 802.15.4 packet loss ratios in curve 1 and curve 2 are approximately 5-7\% less than that in curve 3. Furthermore, comparing curve 1 and curve 2 to curve 6 , where there is collision loss, we still take the case of $\mathrm{x}=40 \mathrm{~dB}$ for instance and see the IEEE 802.15.4 packet loss ratios in curve 1 and curve 2 are approximately $30-35 \%$ less than that in curve 6 . Therefore, in reality, an Rx-to-Tx turnaround time can lead to a significant decline in IEEE 802.15.4 coexistence performance, especially when collision loss exists.

Moreover, the CCA partial detection effect addressed in Sect. 3(2) can also be observed in Fig. 11. In case of $y=212 \mathrm{~dB}$, taking curve 3 for example, we see that in R2, curve 3 shows an "arc" rather than a "1-0" type of right-angle, which exactly reflects the CCA partial detection effect.

\section{Simulation Results}

In Sect. 5, the coexistence performance metrics of IEEE 802.15.4 WSNs under IEEE $802.11 \mathrm{~b} / \mathrm{g}$ interference are given by Eqs. (17), (21) and (22), respectively. Among those 


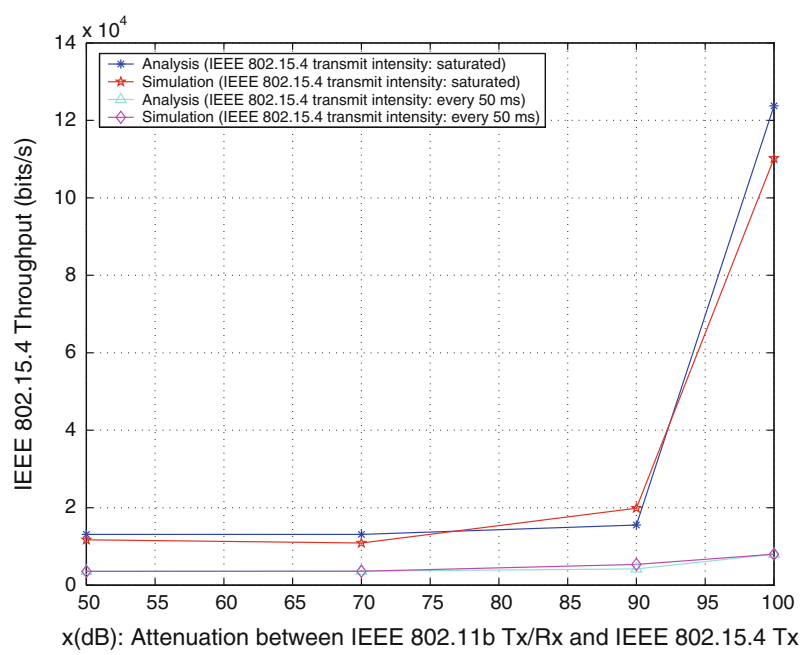

Fig. 13 IEEE 802.15.4 throughput in three coexistence regions under saturated IEEE 802.11b interference

metrics, the analytical packet loss ratio performance has been evaluated by the simulation and the experiments as shown in Fig. 11 where the analysis, simulation and experimental results have a good match. To evaluate the analysis of the other two performance metrics, i.e. throughput and the expected packet delay, we are using only the OPNET simulation both because these two metrics are not able to be achieved directly from our experiment implementation and because the simulation results have proved to have a good match with the experimental ones in Sect. 5.2.1.

Furthermore, we investigate the IEEE 802.15.4 coexistence performance in all the three coexistence regions. We therefore set the attenuation losses between an IEEE $802.11 \mathrm{~b}$ Tx/Rx and an IEEE 802.15.4 Tx are $50 \mathrm{~dB}(\mathrm{R} 1), 70 \mathrm{~dB}(\mathrm{R} 1), 90 \mathrm{~dB}(\mathrm{R} 2)$ and $100 \mathrm{~dB}$ (R3), respectively. For getting good links as assumed in Sect. 5.1, we put the IEEE 802.11b Tx and Rx $1 \mathrm{~m}$ away from each other, and $0.1 \mathrm{~m}$ in between the IEEE 802.15.4 Tx and Rx. Besides, as always assumed in this work, the IEEE $802.11 \mathrm{~b}$ traffic intensity is set as saturated. And the IEEE 802.15.4 traffic intensity is set in two modes: saturated and constant transmission with $50 \mathrm{~ms}$ packet interval time, respectively. The rest of simulation parameters are shown in Table 1. As shown in Figs. 13 and 14, in general, the analytical results have a good match with the simulation ones in all three regions and in both IEEE 802.15.4 transmission modes, which suggests our performance analysis in Sect. 4 is reasonably accurate.

\section{Conclusions}

In this paper, we studied the coexistence performance of IEEE 802.15.4 WSNs under IEEE $802.11 \mathrm{~b} / \mathrm{g}$ interference. By well-designed experiments, our work confirmed that IEEE 802.15.4 WSNs can suffer from heavy IEEE 802.11b/g interference if the channel is not allocated properly. Moreover, we revealed two important implementation factors, i.e., IEEE 802.15.4 Rx-to-Tx turnaround time and CCA partial detection effect, which can have significant impact on IEEE 802.15.4 WSNs coexistence performance in reality, e.g., a long IEEE 802.15.4 Rx-to-Tx turnaround time can impair the CCA performance and therefore 


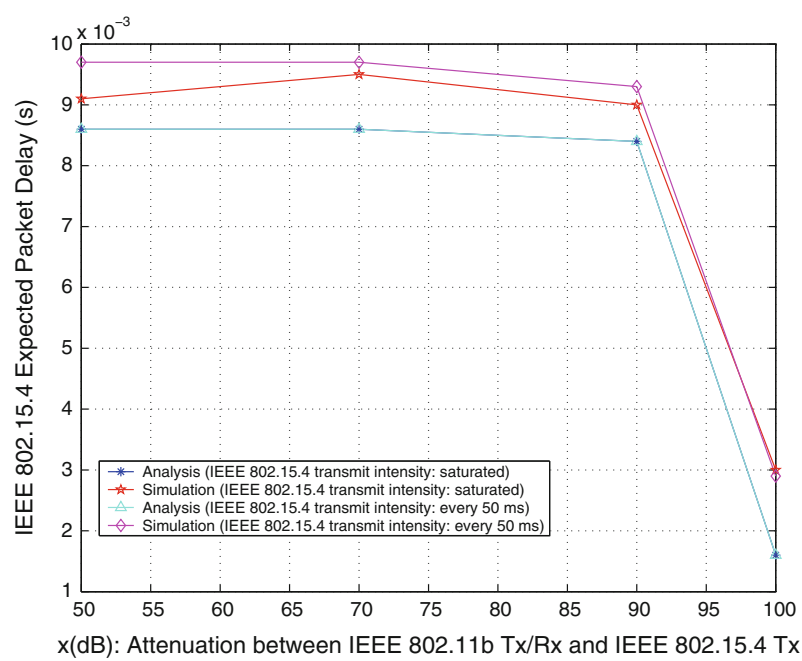

Fig. 14 IEEE 802.15.4 expected packet delay three coexistence regions under saturated IEEE 802.11b interference

the IEEE 802.15.4 WSNs coexistence performance. Taking these implementation factors into account, we improve the analytical coexistence model that we proposed in the previous work. The enhanced model can precisely explain and predict the IEEE 802.15.4 WSNs coexistence performance. Furthermore, under the guidance of the model, the IEEE 802.15.4 WSNs coexistence performance are extensively investigated in all of the three coexistence regions in different scenarios by analysis, simulation and experiments. The simulation and experimental results agree with our analysis. Integrating many insights into the coexistence issue, the model can be helpful in resolving the coexistence issue. One example can be found in [7].

Open Access This article is distributed under the terms of the Creative Commons Attribution Noncommercial License which permits any noncommercial use, distribution, and reproduction in any medium, provided the original author(s) and source are credited.

\section{References}

1. Petrova, M., Riihijarvi, J., Mahonen, P., \& Labella, S. (2006). Performance study of IEEE 802.15.4 using measurements and simulations. In Proceedings of the IEEE WCNC'06, Las Vegas, USA.

2. Howitt, I., \& Gutierrez, J. A. (2003). IEEE 802.15.4 low-rate wireless personal area network coexistence issues. In Proceedings of the IEEE WCNC'03 (Vol. 3, pp. 1481-1486).

3. Shin, S., Park, H., Choi, S., \& Kwon, W. (2005). Packet error rate analysis of IEEE 802.15.4 under IEEE 802.11b interference. In Proceedings of the WWIC'05 (pp. 279-288).

4. Sikora, A. (2005). Coexistence of IEEE 802.15.4 with other systems in the 2.4 GHz-ISM-Band. In Proceedings of the IMTC'05 (Vol. 3, pp. 1786-1791)

5. Yuan, W., Wang, X., \& Linnartz, J. P. M. G. (2007). A Coexistence model of IEEE 802.15.4 and IEEE $802.11 \mathrm{~b} / \mathrm{g}$. In Proceedings of the SCVT'07, Delft, The Netherlands.

6. Yuan, W., Wang, X., Linnartz, J. P. M. G., \& Niemegeers, I. G. M. M. (2010). Experimental validation of a coexistence model of IEEE 802.15.4 and IEEE 802.11b/g networks. International Journal of Distributed Sensor Networks, Article ID 581081, Hindawi. 
7. Yuan, W., Linnartz, J. P. M. G., \& Niemegeers, I. G. M. M. (2010). Adaptive CCA for IEEE 802.15.4 wireless sensor networks to mitigate interference. In IEEE wireless communications and networking conference $(W C N C)$ (pp.1-5). Sydney, Australia.

8. IEEE Std. 802.11 Wireless MAC and PHY Layer Spec. (1999).

9. Ramachandran, I., \& Roy, S. (2007). Clear channel assessment in energy-constrained wideband wireless networks. IEEE Wireless Communications Magazine, 14(3), 70-78.

10. IEEE Standard for Information Technology Part 15.4: Wireless Medium Access Control and Physical Layer Specifications for Low-Rate Wireless Personal Area Networks, IEEE Std. 802.15.4-2006.

11. Gallager, R. G. (1996). Discrete stochastic processes. Dordrecht: Kluwer.

12. Espina, J., Falck, T., \& Mülhens, O. (2006). Network topologies, communication protocols, and standards. In G. Z. Yang (Ed.), Body sensor networks (pp. 145-182). Berlin: Springer.

\section{Author Biographies}
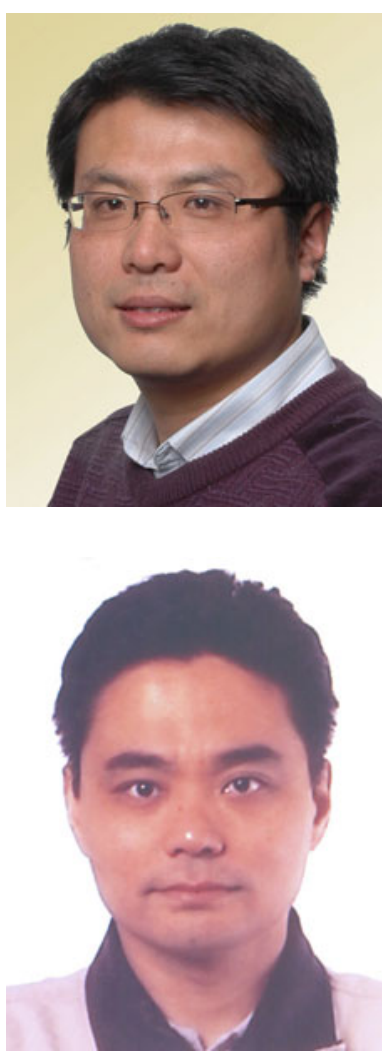

Wei Yuan received M.S. in electrical engineering from Twente University, Enschede, The Netherlands, in 2005. During 2006 to 2010, he was a research scientist with Distributed Sensor Systems group, Philips Research, Eindhoven, The Netherlands. From 2006 upon till now, he is an external Ph.D. student with Wireless and Mobile Communication Group, Delft University of Technology, Delft, The Netherlands. His research interests include wireless sensor networks and broadband access networks.

Xiangyu Wang is currently a senior research scientist at Philips Research Europe. He works in the area of wireless sensor networks and Internet of Things, particularly for lighting control applications. His research interests include networking protocols, and system architecture design. He has authored over 20 patent applications and 2 granted patents. Xiangyu Wang received B.Eng. and M.Eng. degrees from Xi' an Jiaotong University in China in 1993 and 1996 respectively. $\mathrm{He}$ also received a Professional Doctorate in Engineering degree from Technische Universiteit Eindhoven in the Netherlands in 2002. 


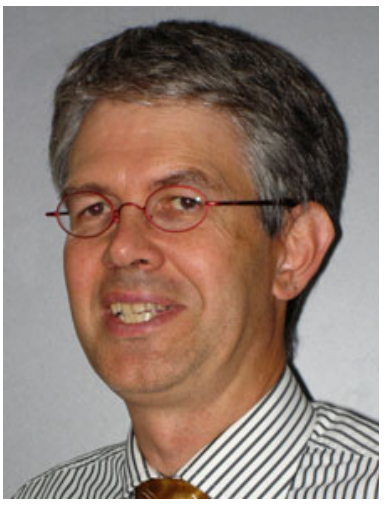

Jean-Paul M. G. Linnartz is a part-time professor at Eindhoven University of Technology in the Signal Processing Systems (SPS) group. As a Senior Director at Philips Research in Eindhoven, the Netherlands, he has led Research groups in the area of Electronic Systems and Silicon Integration, in Information Security and in Connectivity. He joined Philips in 1995. He applied communication signal detection principles in the field of electronic watermarking, and he invented various attacks and security measures. He became Fellow of the IEEE for his work on privacy-preserving methods of biometric identification that prevent misuse of templates from data bases. He proposed algorithms to mitigate Doppler intercarrier interference in OFDM reception for NXP chip sets for mobile DVB television reception. Prof. Linnartz holds more than 30 US patents, some of which are actively used in the industry or in an international standard. In 1992-1993, he was an Assistant Professor at The University of California at Berkeley. In 1993, he introduced Multi-Carrier CDMA. From 1988-1991 and in 1994, he was Assistant and Associate Professor at Delft University of Technology, respectively. He received his Ph.D. (Cum Laude) from T.U. Delft in December 1991 and his M.Sc. (Cum Laude) from Eindhoven University of Technology in 1986.

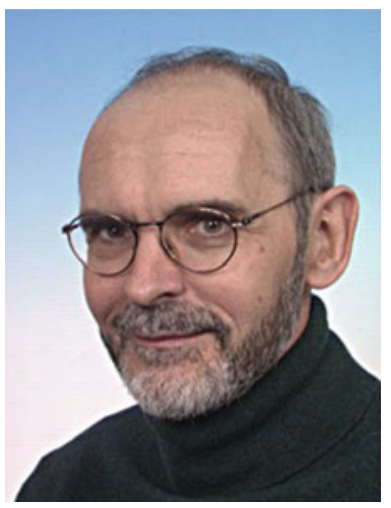

Ignas G. M. M. Niemegeers got a degree in Electrical Engineering from the University of Ghent, Belgium, in 1970. In 1972 he received a M.Sc. degree in Computer Engineering and in 1978 a Ph.D. degree from Purdue University in West Lafayette, Indiana, USA. From 1978 to 1981 he was a designer of packet switching networks at Bell Telephone Mfg. Cy, Antwerp, Belgium. From 1981 to 2002 he was a professor at the Computer Science and the Electrical Engineering Faculties of the University of Twente, Enschede, The Netherlands. From 1995 to 2001 he was Scientific Director of the Centre for Telematics and Information Technology (CTIT) of the University of Twente, a multi-disciplinary research institute on ICT and applications. Since May 2002 he holds the chair Wireless and Mobile Communications at Delft University of Technology, where he is heading the Telecommunications Department. He was involved in many European research projects, e.g., the EU projects MAGNET and MAGNET Beyond on personal networks, EUROPCOM on UWB emergency networks, eSENSE and CRUISE on sensor networks. He is presently involved in iCore, an FP7 project on the Internet-of-Things. He is a member of the Expert group of the European technology platform eMobility, the Dutch Transsectoral Research Academy for Complex Networks and Services and IFIP TC-6 on Networking. His present research interests are future home networks, personal networks, cooperative and cognitive network, in particular aimed at the application domains health care, smart living and energy. He has (co)authored around 300 scientific publications and has coauthored a book on Personal Networks. 